\title{
ANÁLISE DA RELAÇÃO ENTRE A VISÃO DOS PROPRIETÁRIOS SOBRE OS SISTEMAS DE TRABALHO DE ALTO ENVOLVIMENTO (STAE) E SEUS IMPACTOS SOBRE O POTENCIAL DE CRESCIMENTO DAS MICRO E PEQUENAS EMPRESAS
}

\section{ANALYSIS OF THE RELATIONSHIP BETWEEN OWNERS' VIEW ON HIGH INVOLVEMENT WORK SYSTEMS (HIWS) AND THEIR IMPACTS ON THE GROWTH POTENTIAL OF MICRO AND SMALL ENTERPRISES}

Denilson Aparecida Leite Freire

Universidade Nove de Julho

Faculdade de Gestão e Negócios (FAGEN) - Universidade Federal de Uberlândia (UFU)

Uberlândia, MG, Brasil

E-mail: denilson.freire@hotmail.com

\section{RESUMO}

Analisou-se o impacto da visão do proprietário sobre as práticas de gestão de pessoas, focada nos Sistemas de Trabalho de Alto Envolvimento, observando se essas contribuem ou não para o crescimento das MPE's. Trabalhou-se, então, com três variáveis: primeiro, a visão do proprietário, analisada por meio da teoria de identidade organizacional (IO), na percepção deste; em segundo lugar, a adoção de sistemas de trabalho de alto envolvimento (STAE) e, em terceiro, o potencial de crescimento (PC) da empresa. Foi realizado um levantamento com os proprietários de empresas de micro e pequeno porte da região sudeste. Foram obtidas 109 respostas. Os resultados comprovaram que há impacto positivo do caráter normativo de Identidade Organizacional nos Sistemas de Trabalho de Alto Envolvimento e desses sobre o potencial de crescimento das MPE's. Comprovou, também, que há um impacto negativo do caráter utilitarista de IO sobre o STAE e que há impactos da identidade normativa e utilitarista sobre o PC.

Palavras-chave: Gestão Estratégica de Pessoas. Identidade Organizacional. Sistemas de Trabalho de Alto Envolvimento. Potencial de Crescimento. Micro e Pequenas Empresas.

\section{ABSTRACT}

The impact of the owner's view on the practices of people management, focused on the High Involvement Work Systems, was analyzed, observing whether or not these contribute to the growth of MPEs. We then worked with three variables: first, the owner's view, analyzed through the theory of organizational identity (IO), in the perception of it; Secondly, the adoption of highly engaging work systems (STAE) and, thirdly, the company's growth potential (PC). A survey was carried out with owners of micro and small companies in the southeast region. A total of 109 responses were obtained. The results showed that there is a positive impact of the normative character of Organizational Identity in Highly Involved Work Systems and of these on the growth potential of MPEs. It also showed that there is a negative impact of the utilitarian character of IO on STAE and that there are impacts of the normative and utilitarian identity on the $P C$.

Keywords Strategic Management of People. Organizational Identity. Highly Involved Work Systems. Growth potential. Micro and Small Business. 


\section{INTRODUÇÃO}

A estratégia de gestão de pessoas é um dos fatores que impactam no sucesso de longo prazo das organizações (FABI et al., 2009). Em um mercado extremamente competitivo, a Gestão de Pessoas necessita estar alinhada à estratégia da organização (BECKER et al., 2001). Esse alinhamento sofre, normalmente, a influência da percepção e ação dos proprietários e da alta administração das empresas (DAVIDSSON et al., 2009). No caso das empresas micro e pequenas, essa influência é até maior, devido ao envolvimento comum dos proprietários nas ações do dia a dia (TOCHER; RUTHERFORD, 2009).

Sendo assim, o objetivo deste estudo foi analisar qual o impacto da percepção do proprietário das MPEs sobre as práticas de gestão de pessoas adotadas e as influências dessas sobre o potencial de crescimento organizacional.

Em função da complexidade que é definir e pesquisar cada uma dessas dimensões (visão do proprietário, gestão de pessoas e potencial de crescimento), uma vez que, em cada um deles é possível estudar outra multiplicidade de conteúdo, elegeu-se três variáveis que pudessem ser mensuradas.

Para a análise da gestão estratégica de pessoas foi utilizada a variável Sistemas de Trabalho de Alto Envolvimento (STAE). De uma maneira geral, esses sistemas podem ser compreendidos como uma estrutura organizacional que agrupa trabalho, pessoas, tecnologias e informações de maneira a otimizar os resultados organizacionais. Essa inter-relação tem como objetivo maximizar o desempenho em termos de respostas eficazes às exigências do mercado (NADLER et al., 1994).

Para a análise da percepção do proprietário elegeu-se a variável Identidade Organizacional (IO) entendida como uma imagem projetada pela alta direção, por meio das suas crenças e valores (PRATT; FOREMAN, 2000). Uma das influências da visão do proprietário é sobre a estratégia de gestão de pessoas implementada e desenvolvida dentro das organizações, principalmente, nas micro e pequenas empresas, onde a presença dele é maior que nas médias e grandes organizações (DAVIDSSON et al., 2009).

E, finalmente, para a mensuração do crescimento optou-se por analisar o potencial de crescimento das micro e pequenas organizações segundo uma métrica específica. Não se trata de uma tarefa fácil, tal mensuração, já que a maioria das pesquisas utiliza-se dos indicadores econômicos disponibilizados em balanços pelas empresas. Sabe-se, entretanto, que é fato raro a divulgação, ou a permissão, de uso de balanços pelos proprietários das micro e pequenas empresas. Para sanar tal dificuldade, a mensuração foi realizada por meio de métricas de: orientação de crescimento futuro da organização; recursos organizacionais para suportar esse crescimento; e existência da oportunidade de mercado (DAVIDSSON et al., 2009). Tal potencial foi mensurado, então, no cruzamento de variáveis atuais e futuras que consiste na idade atual da organização, no número de empregados atuais (IBGE, 2010) e na percepção do nível de crescimento do proprietário (RUTHERFORD et al., 2004).

O estudo poderá contribuir para uma melhor compreensão das estratégias de gestão de pessoas a serem adotadas por essas empresas em um ambiente competitivo, visando sua sobrevivência e crescimento.

Sabe-se que é crescente a participação das micro e pequenas empresas na estrutura econômica brasileira. Segundo o Anuário do Trabalho das Micros Pequenas e Médias Empresas, Elaborado pelo SEBRAE (Serviço Brasileiro de Apoio à Micro e Pequena Empresa) e pelo Departamento Intersindical de Estatística e Estudo Socioeconômicos (DIEESE), entre 2001 e 2009, o número de donos de micro e pequenas 
empresas (MPE's) e de trabalhadores autônomos passou de 20,2 milhões para 22,9 milhões, demonstrando que é cada vez maior a participação dessas empresas no fortalecimento da economia brasileira.

Apesar do número crescente de MPE's na economia, muitas não conseguem sobreviver aos primeiros anos de existência. Dentre as principais causas de mortalidade das empresas, na opinião dos micro e pequenos empresários estão: a falta de clientes (18\%), a falta de capital $(10 \%)$ e problemas relacionados com a administração e o planejamento (10\%), evidenciando que os dois primeiros fatores estão intrinsecamente relacionados com o terceiro (SEBRAE/SP, 2010).

Para que haja a manutenção desse crescimento, aponta a pesquisa que, cerca de $75 \%$ dos pequenos empresários, enfatizaram a relevância da Gestão de Pessoas como um fator importante e, ao mesmo tempo, um desafio a ser superado pelas empresas, uma vez que, apenas $58,1 \%$ das Pequenas e Médias Empresas (PMEs) afirmaram possuir um setor voltado para as práticas de Gestão de Pessoas (FAEDPYME, 2009). Então, em última instância, a visão dos proprietários pode ser o determinante de uma estratégia de gestão de pessoas que irá contribuir, ou não, para o crescimento da organização.

\section{REFERENCIAL TEÓRICO}

\section{Sistemas de Trabalho de Alto Envolvimento (STAE)}

A partir dos anos 80 , e principalmente, durante a década de 90 , o mundo passa por grandes transformações econômicas, tornando as relações comerciais e de trabalho cada vez mais globais e competitivas, exigindo das organizações ações cada vez mais estratégicas (RUAS, 1995). A Gestão Estratégica de Pessoas visa, assim, assessorar a organização no alcance dos seus objetivos, devendo, para isso, caminhar junto ao planejamento estratégico da organização. Nessa era, sua função é de subsidiar a alta direção nos assuntos relacionados como a qualidade dos talentos da organização, no desenvolvimento desse e da organização e das políticas de manutenção dos Recursos Humanos (ALBUQUERQUE, 2002).

Surge, a partir desse momento, várias vertentes e estudos sobre a Gestão Estratégica de Pessoas. Os estudos atuais apresentam, na sua grande maioria, uma dicotomomia entre os constructos que estruturam a visão estratégica de pessoas. Uma delas diz respeito aos modelos Hard e Soft Elaborados por Storey (1986) e outra diferenciando os modelos de controle e de comprometimento propostos por Walton (1985). No modelo Hard, os recursos humanos são objeto de um planejamento formal, sendo vistos como um fator de produção conjuntamente com a propriedade e capital (LEGGE, 1995). Além disso, o modelo enfatiza os aspectos estratégicos quantitativos e de negócio, na GRH. Gerem os RH de um modo "racional", tal como estes fossem outro fator econômico qualquer (STOREY, 1987). Já o modelo Soft foca na construção, em longo prazo, da vantagem competitiva das organizações por meio de uma força de trabalho flexível de alta qualidade e comprometida. A ênfase está em obter respostas positivas dos colaboradores por meio da comunicação adequada e técnicas motivacionais e estilo de liderança (STOREY, 1987).

Silva et al. (2009), baseando-se no trabalho de Walton (1985), relacionaram esses conceitos a outras duas estratégias da gestão de pessoas: controle e comprometimento. Na estratégia de controle, os empregados são vistos como números, como custo, como mais um fator de produção que, para desempenharem, devem ser controlados. Neles, a gestão de pessoas é caracterizada por uma divisão do 
trabalho em pequenas unidades fixas nas quais os indivíduos eram responsáveis, mas sobre o controle direto dos seus supervisores. Este tipo de sistema tem por objetivo reduzir os custos diretos do trabalho ou melhorar a eficiência, pela imposição de padrões de conformidade (WALTON, 1985). Essa estratégia pode ser relacionada à visão Hard de gestão proposta por Storey (1987).

Já as estratégias de compromisso visam estabelecer ligações entre os objetivos organizacionais e pessoais, por meio do envolvimento dos colaboradores, semelhante ao que ocorre na estratégia Soft de Storey (1987). Nesse sistema não há necessidade de controle direto dos supervisores (WALTON, 1985),

A partir da década 80, com a emergência dos tópicos de gestão estratégia e de competitividade das organizações, o que impulsionou a mudança do paradigma de controle para o de comprometimento na gestão de pessoas, a integração entre esses dois modelos passou a ser denominado de Sistemas de Trabalho de Alto Envolvimento (STAE).

Não há uma definição clara do que seja Sistemas de Trabalho de Alto Envolvimento na literatura e, nem mesmo, um acordo sobre as práticas que devam ser incorporadas a esses sistemas (LLOYD; PAYNE, 2004). Apesar dos muitos termos e conceitos, todos levam a uma mesma ideia: de que a participação dos trabalhadores para decidir como realizar o trabalho é benéfico e deve ser estimulado por meio de planos de formação e de remuneração que irão motivá-los a serem mais envolvidos no trabalho (DOODY, 2007).

De uma maneira geral, os Sistemas de Trabalho de Alto Envolvimento (STAE) podem ser entendidos como uma estrutura organizacional que congrega trabalho, pessoas, tecnologias e informações de maneira a otimizar a congruência e a adequação entre elas. Essa inter-relação tem a finalidade de produzir alto desempenho em termos de respostas eficientes às exigências dos clientes e de outras demandas ou oportunidades detectadas no ambiente externo (NADLER et al., 1994).

Não há, entre os autores, também uma unanimidade sobre quais fatores devem constituir os STAE. Dentre as muitas propostas, optou-se pelo modelo proposto por Ciavarella (2003), que estrutura os Sistemas de Trabalho de Alto Envolvimento como aqueles que possuem quatro dimensões: poder, informação, recompensas e conhecimento:

- Poder significa dar aos empregados mais autoridade no processo de tomada de decisões, emergindo, daí, um processo coletivo, acreditando-se que se o colaborador tiver mais autonomia nessas decisões poderia levar a uma maior motivação e envolvimento com o trabalho (DOODY, 2007).

- A informação refere-se ao compartilhamento dessas com todos os funcionários em relação aos plano e metas da organização. O fornecimento de informação aos trabalhadores não só é necessário para a construção de uma base sólida para os níveis mais elevados de poder e conhecimento na organização, como também leva a uma maior qualidade de decisões e sugestões de como os processos de trabalho podem ser melhorados (CIAVARELLA, 2003).

- Recompensas são projetadas para guiarem o desempenho dos funcionários por meio da partilha dos ganhos subsequentes (LAWLER, 1990). É essencial que essas recompensas estejam intimamente ligadas ao desempenho para que os empregados sejam motivados a melhorar os processos de trabalho (CIAVARELLA, 2003).

- Conhecimento se concentra no desenvolvimento de habilidades dos funcionários para que eles possam eficazmente realizar suas tarefas, levando à saída de alta qualidade. Obviamente, os 
funcionários não sendo treinados com as habilidades necessárias para executar no trabalho vai minimizar a probabilidade de realizarem em ou acima das expectativas (CIAVARELLA, 2003).

Os Sistemas de Trabalho de Alto Envolvimento podem ser comparados ao modelo Soft de Gestão Estratégica de Pessoas, que por sua vez, possuem fundamentação nas estratégias de comprometimento, justificando-se, assim, o uso do STAE como construto de mensuração do nível de Gestão Estratégica de Pessoas.

\section{Identidade Organizacional}

A maioria das pesquisas sobre identidade organizacional baseia-se no artigo seminal de Albert e Whetten (1985), intitulado de Organization Identity. Esses autores definiam a identidade como sendo o elemento central, distintivo e duradouro de uma organização.

Para esses autores, a Identidade é composta por três elementos chaves: centralidade, distinção e continuidade temporal. Centralidade está relacionada à identificação das características essenciais da organização; a distinção procura destacar os elementos que diferenciam uma organização de outras, que possuem características similares e que permitam a comparação; e finalmente, a continuidade temporal visa destacar as características que dão estabilidade à organização ao longo do tempo (ALBERT; WHETTEN, 1985).

As definições dos construtos que devam fazer parte da medida da identidade organizacional foram proposta por Albert e Whetten (1985). Para eles, as organizações podem possuir dois sistemas de valores, um normativo, enfatizando as tradições, os símbolos e a preservação de uma ideologia; e outro utilitário, focado na racionalidade econômica e na maximização dos lucros. Podem existir esses dois sistemas simultaneamente na mesma organização, denominando-se de organizações híbridas (ALBERT; WHETTEN, 1985).

A organização é tida utilitária quando os membros percebem que o foco da organização está orientado, principalmente para os fatores econômicos. A identidade é mais normativa quando esses membros percebem que as principais diretrizes da organização está orientada, principalmente, para as preocupações ideológicas e baseada em valores e crenças (GIOIA; THOMAS, 1996).

\section{Potencial de Crescimento}

Há poucas pesquisas, no Brasil, sobre as estratégias de crescimento utilizadas pelas pequenas e médias empresas (PME), constituindo-se, assim, em uma oportunidade de estudo (LIMA, 2010). Quando se fala em crescimento organizacional, o senso comum pode levar a pensar esse construto como um sinônimo de desempenho organizacional. Essa concepção, entretanto, é errônea e há diferenças entre crescimento e desempenho (COMBS et al., 2005).

O desempenho é uma variável muito utilizada nas pesquisas em administração, contudo, sua definição e operacionalização não são tão simples. A principal dificuldade é que o desempenho tem um caráter multidimensional, ou seja, ela não possui apenas um construto que o represente, mas vários (COMBS 
et al., 2005). O desempenho, na perspectiva da muldimensionalidade, pode ser analisado sobre três domínios com graus de abrangência crescentes: o desempenho financeiro, que ampliado constituiria o desempenho operacional e o somatório desses dois, culminariam na eficácia organizacional (VENKATRAMAN; RAMANUJAM, 1986).

Mais, ainda assim, a análise do desempenho financeiro deveria se dar a partir de três variáveis: Lucratividade, crescimento e noção de valor de mercado (COMBS et al., 2005). A lucratividade pode ser mensurada pela apuração do lucro líquido sobre o faturamento bruto e é o construto mais utilizado para mensurar desempenho. $O$ crescimento, geralmente pode ser mensurado pela variação da receita líquida, crescimento do número de funcionários ou crescimento dos ativos totais (COMBS et al., 2005).

Nas pesquisas acadêmicas, nem sempre as empresas dispõem-se facilmente dos seus dados financeiros, entretanto, a grande maioria disponibiliza o número de funcionários, tornando, essa métrica mais fácil de ser apurada na mensuração do crescimento organizacional.

No Brasil, órgãos como o Sebrae e o IBGE, utilizam-se, além dos dados financeiros, da métrica de número de funcionários para classificar o nível de crescimento das empresas. Sendo assim, as empresas de alto crescimento seriam aquelas que apresentassem um crescimento médio do pessoal ocupado assalariado maior que $20 \%$ ao ano, por um período de três anos, e que tivessem pelo menos 10 pessoas assalariadas no ano inicial de observação. As empresas de médio crescimento seriam aquelas que apresentaram crescimento de pessoal assalariado maior que $5 \%$ e até $20,0 \%$ ao ano e as empresas de baixo crescimento aquelas que demonstrassem crescimento até $5 \%$ ao ano (IBGE, 2010).

Um grande desafio é, portanto, a mensuração do nível de crescimento das organizações, pois as várias tentativas feitas não têm sido bem sucedidas (PASANEN, 2006). Uma das possíveis razões é a falta de critérios únicos para se medir e comparar as empresas em diferentes locais do mundo.

Rutherford et al. (2004) estruturaram uma escala baseada na percepção de crescimento ou não pelos proprietários, pela organização, no último ano trabalhado por eles. Uma maneira, então, de se mensurar o crescimento das empresas consiste no cruzamento de variáveis atuais, como o tempo de vida da empresa, a variação da receita líquida e o número de funcionários atuais (IBGE, 2010); e futuras como a percepção do nível de crescimento do proprietário (RUTHERFORD et al., 2004). 


\section{METODOLOGIA}

O relacionamento entre as questões de pesquisa e suas respectivas hipóteses pode ser mais bem organizado esquematicamente, conforme demonstrado na Figura 1 e que representa o modelo Conceitual de pesquisa.

Figura 1 - Modelo Conceitual de Pesquisa

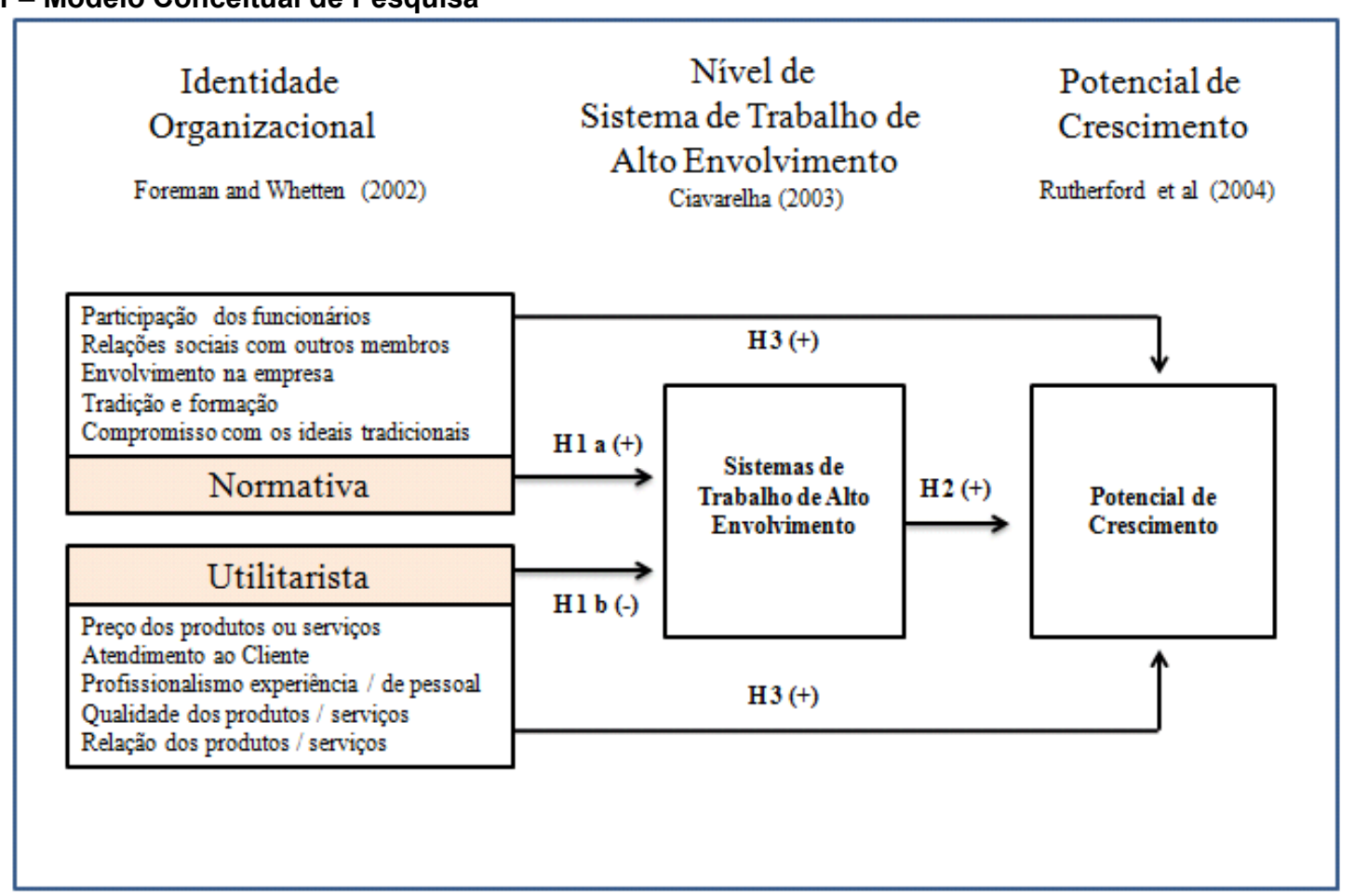

Fonte: elaborada pelo autor.

O modelo pressupõe que a visão dos empresários, traduzida em uma identidade do tipo normativa, tende a levar à implementação de um maior nível de estratégias de Gestão de Pessoas que promovam o alto envolvimento dos seus colaboradores $\left[\mathrm{H}_{1 \mathrm{a}}(+)\right]$ e, consequentemente, esses sistemas impactam positivamente no maior potencial de crescimento organizacional $\left[\mathrm{H}_{2}(+)\right]$. Caso, essas afirmativas se confirmem, traduz-se, ainda que, uma identidade normativa pode levar a maiores níveis de desenvolvimento da empresa $\left[\mathrm{H}_{3}(+)\right]$.

Como contraponto, o modelo demonstra que a visão dos empresários, traduzida em uma identidade do tipo utilitarista, tende a levar à implementação de baixas práticas de Gestão de Pessoas que promovam o alto envolvimento dos seus colaboradores $\left[\mathrm{H}_{1 \mathrm{~b}}(+)\right]$ e, consequentemente, esses sistemas impactam negativamente no maior potencial de crescimento organizacional. Caso, essas afirmativas se confirmem, traduz-se, ainda que, uma identidade do tipo utilitarista pode levar a menores níveis de desenvolvimento da empresa $\left[\mathrm{H}_{3}(-)\right]$.

A investigação teve o caráter quantitativo (COUTINHO, 2004). Para levantamento de dados, propôsse uma Survey como principal estratégia de pesquisa voltada à comprovação das hipóteses (PINSONNEAULT; KRAEMER, 1993). O universo de pesquisa foi composto por 3.108.608 empresas de micro e pequeno porte da Região Sudeste brasileira (SEBRAE; DIEESE, 2010). A amostra foi definida utilizando-se dos critérios mínimos exigidos pela técnica estatística de modelagem de equações estruturais. 
Essa técnica permite avaliar o impacto de uma variável sobre a outra que é o objetivo dessa pesquisa: analisar o impacto da IO sobre os STAE's e destes sobre o potencial de crescimento das organizações. Não há, na literatura, uma uniformidade em relação ao número de respondentes mínimos na composição amostral para modelagem. Podem ser encontradas indicações de valores variando entre 100 e 200 casos de acordo com o Software a ser utilizado (BIDO et al., 2009). Baseando-se nessas ponderações e no número de questões propostas para essa pesquisa a amostra foi de, no mínimo, 100 casos, sendo alcançadas 109 respostas válidas.

Para a elaboração do questionário final de pesquisa foram adaptadas as escalas de Identidade Organizacional, Sistemas de Trabalho de Alto Envolvimento e Potencial de Crescimento devido às particularidades do cenário econômico e social das micro e pequenas empresas (FREIRE, 2013).

A técnica estatística escolhida para a análise dos dados baseou-se em uma Modelagem em Equações Estruturais (MEE) com estimação PLS, utilizando-se do software SMART PLS 2.0 (RINGLE; WILL, 2005). Essa técnica estatística está baseada no método dos Mínimos Quadrados Parciais PLS-PM (Partial Least Squares Path Modeling). Utilizou-se, no PLS-PM, a técnica de reamostragem denominada de Bootstrapping, na qual os dados originais são repetidamente amostrados com substituição para estimação do modelo. Essa técnica apresenta os resultados do teste da distribuição $t$ Student para várias amostras. De acordo com o valor de $t$ pode-se definir se os coeficientes padronizados (path coefficients) são significantes (BIDO et al., 2009).

No Bootstrapping, considera-se a hipótese nula $(\mathrm{H} 0)$ de que os coeficientes padronizados sejam iguais a zero. O resultado do $t$ de Student superior a 1,96 indica que há menos de $5 \%$ de rejeitar a $\mathrm{H} 0$. Se essa hipótese for rejeitada indica que a correlação é significante (BIDO et al., 2009).

A aplicação da MEE com estimação PLS-PM traduz-se em uma Análise Fatorial Confirmatória onde foi avaliada a validade dos construtos e as relações entre esses. Para testar a validade foram consideradas três critérios: convergência, discriminância e nomologicidade (HAIR Jr. et al., 2005). O critério de convergência avalia o grau com que duas medidas de um mesmo construto estão correlacionadas. Uma alta correlação indica que o instrumento mensura o que realmente foi esperado em relação ao objeto de estudo. O critério de discriminância avalia o grau com que duas variáveis similares são distintas. Nela, uma baixa correlação entre as variáveis indica que o instrumento é capaz de diferenciar objetos de estudos similares. E, por fim, o critério nomológico avalia o grau com que o instrumento de um objeto de estudo prediz de forma eficaz outros objetos de estudo (HAIR Jr. et al., 2005). Esses critérios estão descritos no Quadro 1, baseandose nas orientações de Ringle e Will (2005) e de Hair Jr. et al. (2005).

\section{Quadro 1 - Critérios de Convergência e Divergência na Estimação PLS-PM}

\begin{tabular}{|lll|}
\hline \multicolumn{1}{|c|}{ Critérios } & \multicolumn{1}{c|}{ Testes } & Referência \\
\hline \multirow{4}{*}{ Convergente } & Variância Extraída (AVE) & Superior a 0,500 \\
& $\begin{array}{l}\text { Alfa de Crombach } \\
\text { Cargas Fatoriais } \\
\text { Confiabilidade Composta }\end{array}$ & $\begin{array}{l}\text { Superior a 0,600 } \\
\text { Superior a 0,500 } \\
\text { Superior a 0,700 }\end{array}$ \\
\hline Divergente & Raiz Quadrada da AVE & $\begin{array}{l}\text { Cargas fatoriais mais altas nas variáveis latentes } \\
\text { em relação às outras. }\end{array}$ \\
\hline Nomológico & $p$-valor do Bootstrapping & Superior a 1,962 \\
\hline
\end{tabular}

Fonte: elaborado pelo autor baseado em Hair Jr. et al. (2005). 
$\mathrm{Na}$ validade discriminante, os indicadores devem possuir cargas fatoriais mais altas nas suas respectivas variáveis latentes (raiz quadrada da AVE) do que em relação às outras variáveis latentes (BIDO et al., 2009). Para definição do tamanho do efeito entre as variáveis latentes utilizou-se da classificação de Cohen (1977) que considera, na análise, o campo das ciências humanas em que as correlações estão sendo usadas. Para ele, explicar $30 \%$ da variância da variável dependente já é considerado um efeito forte.

O fato é que o estado de desenvolvimento de muito da ciência comportamental é tal que não muito da variância da variável dependente é explicável. Isto é essencialmente outro modo de dizer o óbvio: que a ciência comportamental, coletivamente, não é tão avançada como as ciências físicas. Na última, nós podemos frequentemente explicar mais de $99 \%$ da variância da variável dependente, por exemplo, na mecânica clássica (COHEN, 1977, p. 78).

O tamanho do efeito foi mensurado com as seguintes métricas (COHEN, 1977):

- Pequeno: $r=0,100$;

- $\quad$ Médio: $r=0,300$;

- Grande $r=0,500$;

Utilizou-se do modelo reflexivo, isso é, as variáveis latentes IO, STAE e PC são refletidas por indicadores observáveis (modelo reflexivo) e avaliando a relação entre IO sobre STAE; de STAE sobre PC e de $1 \mathrm{O}$ sobre $\mathrm{PC}$, contribuirão para as respostas às hipóteses desta pesquisa.

\section{RESULTADOS E ANÁLISES}

Após executar o algoritmo PLS com a configuração Factor Weighting Scheme, as cargas fatoriais apuradas estão representadas na Figura 2.

Figura 2 - Estimação PLS das Variáveis Latentes IO, STAE e PC

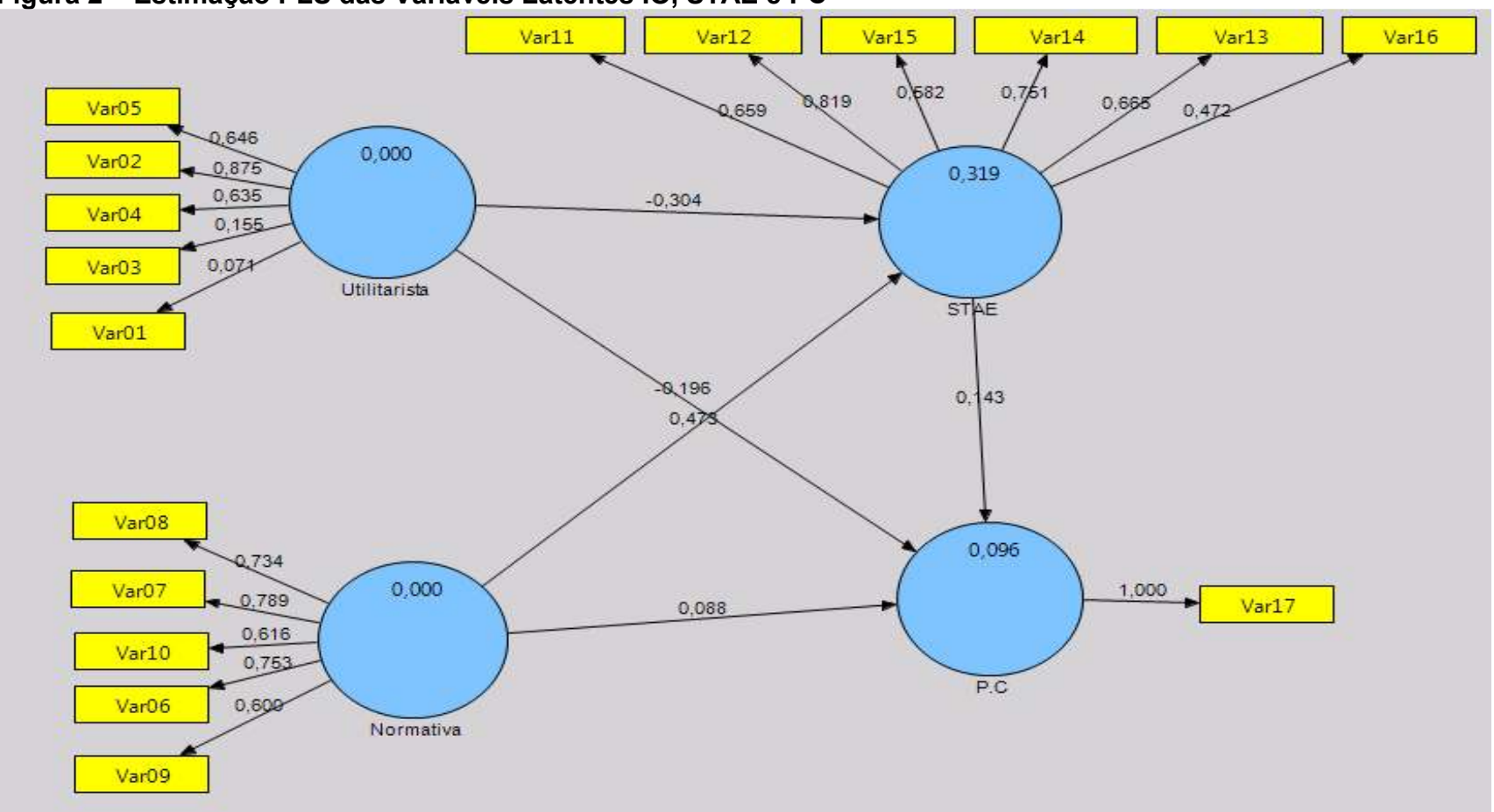

Fonte: Smart PLS 2.0 
Analisando-se as cargas fatoriais das variáveis que compõem os construtos de IO e STAE, observase, na Tabela 1, que três variáveis não obtiveram uma carga fatorial acima de 0,500 , sendo elas:

- Duas delas no construto utilitarista de IO: Var01 - "Oferecer o menor preço possível dos produtos/serviços" obteve carga fatorial igual a 0,071 e Var 03 - "Investir intensamente na qualificação dos profissionais da empresa" com carga fatorial igual a 0,155. Esses dados revelam que essas variáveis não são adequadas para compor o construto utilitarista.

- Uma no construto STAE: Var16 - "Tem seus salários, treinamentos e métodos de seleção decididos pelos sócios ou diretores da empresa diretamente?" com carga fatorial igual a 0,472.

Tabela 1 - Cargas Fatoriais das Variáveis que compõem o Modelo Estrutural

\begin{tabular}{|c|c|c|}
\hline Variável & Descrição da Variável & Carga Fatorial \\
\hline Var01 & Oferecer o menor preço possível dos produtos/serviços & 0,071 \\
\hline Var02 & Aprimorar ainda mais o serviço ao cliente & 0,875 \\
\hline Var03 & Investir intensamente na qualificação dos profissionais da empresa & 0,155 \\
\hline Var04 & $\begin{array}{l}\text { Dispor da melhor qualidade disponível no mercado para os seus } \\
\text { produtos/serviços }\end{array}$ & 0,635 \\
\hline Var05 & Agregar mais inovação aos produtos/serviços & 0,648 \\
\hline Var06 & Maximizar a participação dos funcionários/colaboradores nas decisões & 0,753 \\
\hline Var07 & $\begin{array}{l}\text { Garantir os melhores níveis de bons relacionamentos entre os colaboradores da } \\
\text { empresa }\end{array}$ & 0,789 \\
\hline Var08 & Envolver intensamente a comunidade na estratégia da empresa & 0,734 \\
\hline Var09 & Responsabilizar pelo nível de educação dos colaboradores & 0,609 \\
\hline Var10 & Manter a empresa fiel aos seus objetivos, visão e valores organizacionais & 0,616 \\
\hline Var11 & $\begin{array}{l}\text {... participa de alguma reunião ou programa para discutir melhorias } \\
\text { organizacionais? }\end{array}$ & 0,659 \\
\hline Var12 & $\begin{array}{l}\text {... participa de reuniões periódicas com os sócios ou diretores, para informa-los } \\
\text { dos desenvolvimentos na empresa? }\end{array}$ & 0,812 \\
\hline Var13 & ... recebe salário variável conforme seu desempenho (metas)? & 0,665 \\
\hline Var14 & ... recebe bônus coletivos por trabalharem em equipe ou lucro da empresa? & 0,751 \\
\hline Var15 & ... podem exercer tarefas que vão além do seu cargo oficial, com flexibilidade? & 0,582 \\
\hline Var16 & $\begin{array}{l}\text {... tem seus salários, treinamentos e métodos de seleção decididos pelos sócios } \\
\text { ou diretores da empresa diretamente? }\end{array}$ & 0,472 \\
\hline Var17 & Variação do \% de Crescimento & 1,000 \\
\hline
\end{tabular}

Fonte: elaborada pelo autor com base em resultados do processamento no Smart PLS 2.0

Todas as demais variáveis obtiveram cargas fatoriais acima de 0,500 demonstrando estarem adequadas aos construtos do modelo estrutural. A análise da validade convergente obedeceu aos seguintes critérios: alfa de Crombach maior que 0,600; AVE (Average Variance Extracted) superior a 0,500 e Confiabilidade Composta maior que 0,700 . Os resultados estão expressos na Tabela 2. 
Tabela 2 - Indicadores de Validade Convergente

\begin{tabular}{lccc}
\hline \multicolumn{1}{c}{ Construtos } & Confiabilidade Composta & Alfa de Crombach & AVE \\
\hline Utilitarista & 0,627 & 0,614 & 0,324 \\
Normativo & 0,829 & 0,733 & 0,496 \\
STAE & 0,827 & 0,742 & 0,450 \\
\hline
\end{tabular}

Fonte: elaborada pelo autor baseado nos resultados do SmartPLS 2.0

O Construto normativo atende a todas as exigências de critério, demonstrando possuir validade convergente. No construto STAE e aceita-se o valor de AVE de 0,450 em função do atendimento aos outros dois critérios: alfa de Crombach $(0,742)$ e confiabilidade composta $(0,827)$. O construto utilitarista atende apenas ao critério do alfa de Crombach $(0,614)$, entretanto não corresponde aos exigidos pela confiabilidade composta e para a AVE.

No intuito de melhorar a validade para o construto utilitarista, optou-se por retirar as duas variáveis (Var01 e Var03) do construto utilitarista do modelo estrutural. As cargas fatoriais foram novamente recalculadas e os resultados encontram-se expressos na Figura 3.

Figura 3 - Análise PLS do Modelo Estruturado com Ajuste do Construto Utilitarista

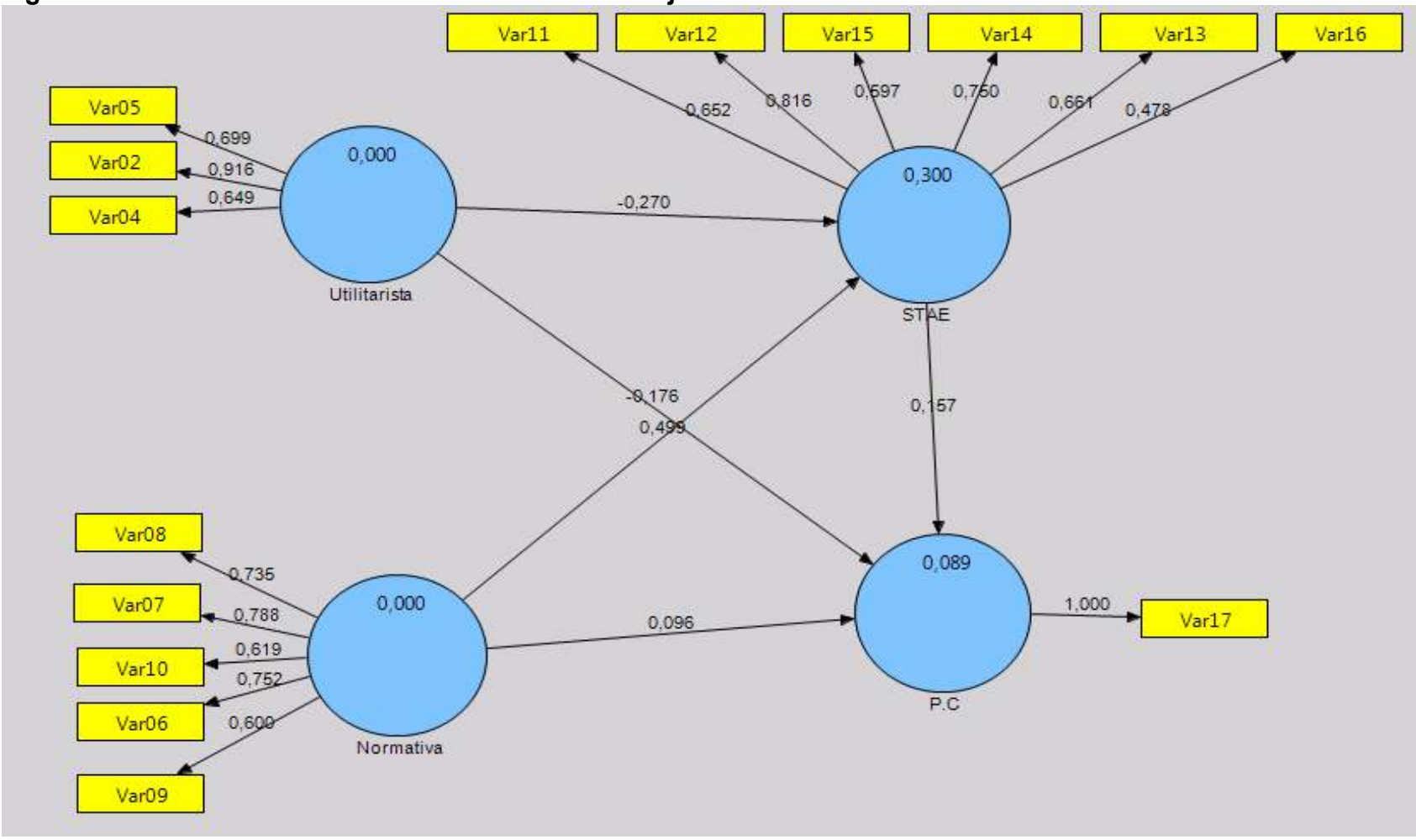

Fonte: Smart PLS 2.0

Analisando-se, agora, as variáveis que compõem os construtos de IO e STAE, observa-se na Figura 4 que todas as variáveis possuem, agora, valores próximos ou superiores a 0,500. A validade convergente confirma que o construto utilitarista passa a ter uma maior consistência, já que os valores para os critérios exigidos são ultrapassados, isto é, obteve-se uma confiabilidade composta de 0,804 (maior que 0,700); alfa 
de Crombach de 0,710 (maior que 0,600) e AVE de 0,583 (maior que 0,500). Vide destaque em negrito na Tabela 3.

Tabela 1 - Indicadores de Validade Convergente no Ajuste do Construto Utilitarista

\begin{tabular}{lccc}
\multicolumn{1}{c}{ Construtos } & Confiabilidade Composta & Alfa de Crombach & AVE \\
\hline Utilitarista & $\mathbf{0 , 8 0 4}$ & $\mathbf{0 , 7 1 2}$ & $\mathbf{0 , 5 8 3}$ \\
Normativo & 0,829 & 0,733 & 0,496 \\
STAE & 0,827 & 0,742 & 0,450 \\
\hline
\end{tabular}

Fonte: Elaborado pelo autor baseado nos resultados do SmartPLS 2.0

$\mathrm{Na}$ análise discriminante, se a raiz quadrada da AVE das variáveis latentes for maior que as correlações entre as demais variáveis, quer dizer que há validade discriminante. A Tabela 4 apresenta as cargas fatoriais cruzadas entre as variáveis e, na interseção, a raiz quadrada da AVE.

\section{Tabela 4 - Análise Discriminante}

\begin{tabular}{|c|c|c|c|}
\hline Construtos & Utilitarista & Normativo & STAE \\
\hline Utilitarista & 0,7635 & & \\
\hline Normativo & 0,0793 & 0,7040 & \\
\hline STAE & $-0,2321$ & 0,4774 & 0,6707 \\
\hline
\end{tabular}

Fonte: Elaborado pelo autor baseando nos dados do Smart-PLS

Observa-se, na Tabela 4, que os valores da raiz quadrada de AVE (em negrito) são maiores que as correlações entre as demais variáveis, indicando que há validade discriminante para o modelo estrutrural. Para finalizar a AFC, foi utilizou-se da técnica Bootstrapping, cujos dados estão representados na Figura 4.

Figura 4 - Análise Booststrapping

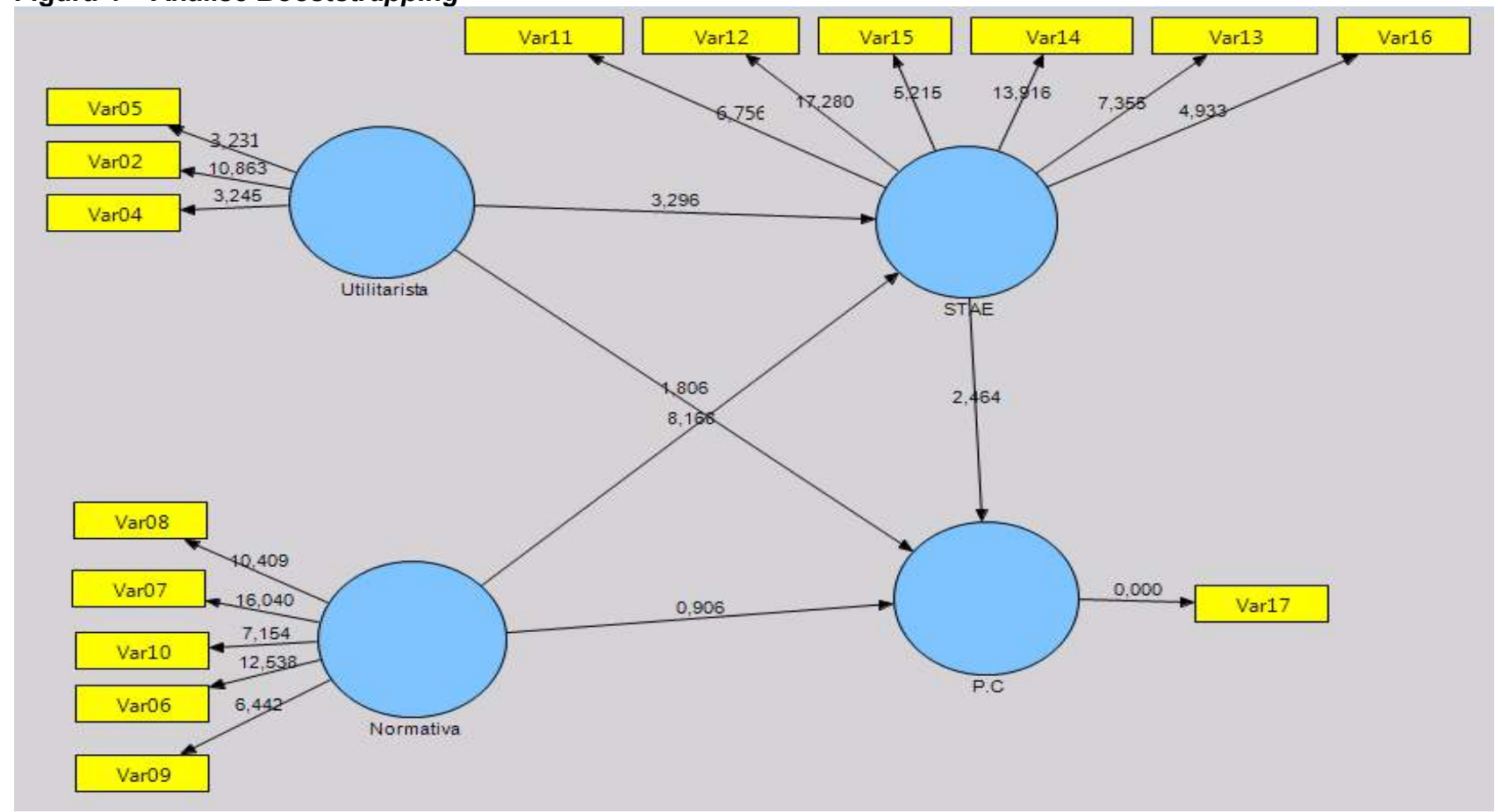

Fonte: Smart PLS 2.0 
Observando os resultados os valores t (ou p-value) calculados por Bootstrap (Tabela 5) apurou-se que todos índices foram maiores que 1,962, revelando que as variáveis são significantes na composição dos construtos.

Tabela 5 - Teste Bootstrapping das Variáveis que Compõem os Construtos

\begin{tabular}{|c|c|c|}
\hline Variável & Descrição da Variável & p-Valor \\
\hline Var02 & Aprimorar ainda mais o serviço ao cliente & 10,863 \\
\hline Var04 & $\begin{array}{l}\text { Dispor da melhor qualidade disponível no mercado para os seus } \\
\text { produtos/serviços }\end{array}$ & 3,245 \\
\hline Var05 & Agregar mais inovação aos produtos/serviços & 3,231 \\
\hline Var06 & Maximizar a participação dos funcionários/colaboradores nas decisões & 12,538 \\
\hline Var07 & $\begin{array}{l}\text { Garantir os melhores níveis de bons relacionamentos entre os colaboradores da } \\
\text { empresa }\end{array}$ & 16,040 \\
\hline Var08 & Envolver intensamente a comunidade na estratégia da empresa & 10,409 \\
\hline Var09 & Responsabilizar pelo nível de educação dos colaboradores & 6,442 \\
\hline Var10 & Manter a empresa fiel aos seus objetivos, visão e valores organizacionais & 7,154 \\
\hline Var11 & $\begin{array}{l}\text {... participa de alguma reunião ou programa para discutir melhorias } \\
\text { organizacionais? }\end{array}$ & 6,756 \\
\hline Var12 & $\begin{array}{l}\text {... participa de reuniões periódicas com os sócios ou diretores, para informa-los } \\
\text { dos desenvolvimentos na empresa? }\end{array}$ & 17,280 \\
\hline Var13 & ... recebe salário variável conforme seu desempenho (metas)? & 7,355 \\
\hline Var14 & ... recebe bônus coletivos por trabalharem em equipe ou lucro da empresa? & 13,916 \\
\hline Var15 & ... podem exercer tarefas que vão além do seu cargo oficial, com flexibilidade? & 5,215 \\
\hline Var16 & $\begin{array}{l}\text {... tem seus salários, treinamentos e métodos de seleção decididos pelos sócios } \\
\text { ou diretores da empresa diretamente? }\end{array}$ & 4,933 \\
\hline
\end{tabular}

Fonte: elaborada pelo autor com base em resultados do processamento no Smart PLS 2.0

Analisadas as variáveis que compõem cada construto, fez-se, a seguir, uma análise das influências e correlações entre as variáveis latentes ou construtos. Os fatores que têm mais relevância no modelo são aqueles que apresentam maiores coeficientes padronizados e, portanto, maior explicação $\left(R^{2}\right)$ da variância (e que está no centro dos círculos de cada variável latente no PLS) como demonstrado na Figura 5. 
Figura 5 - Influências entre as Variáveis Latentes

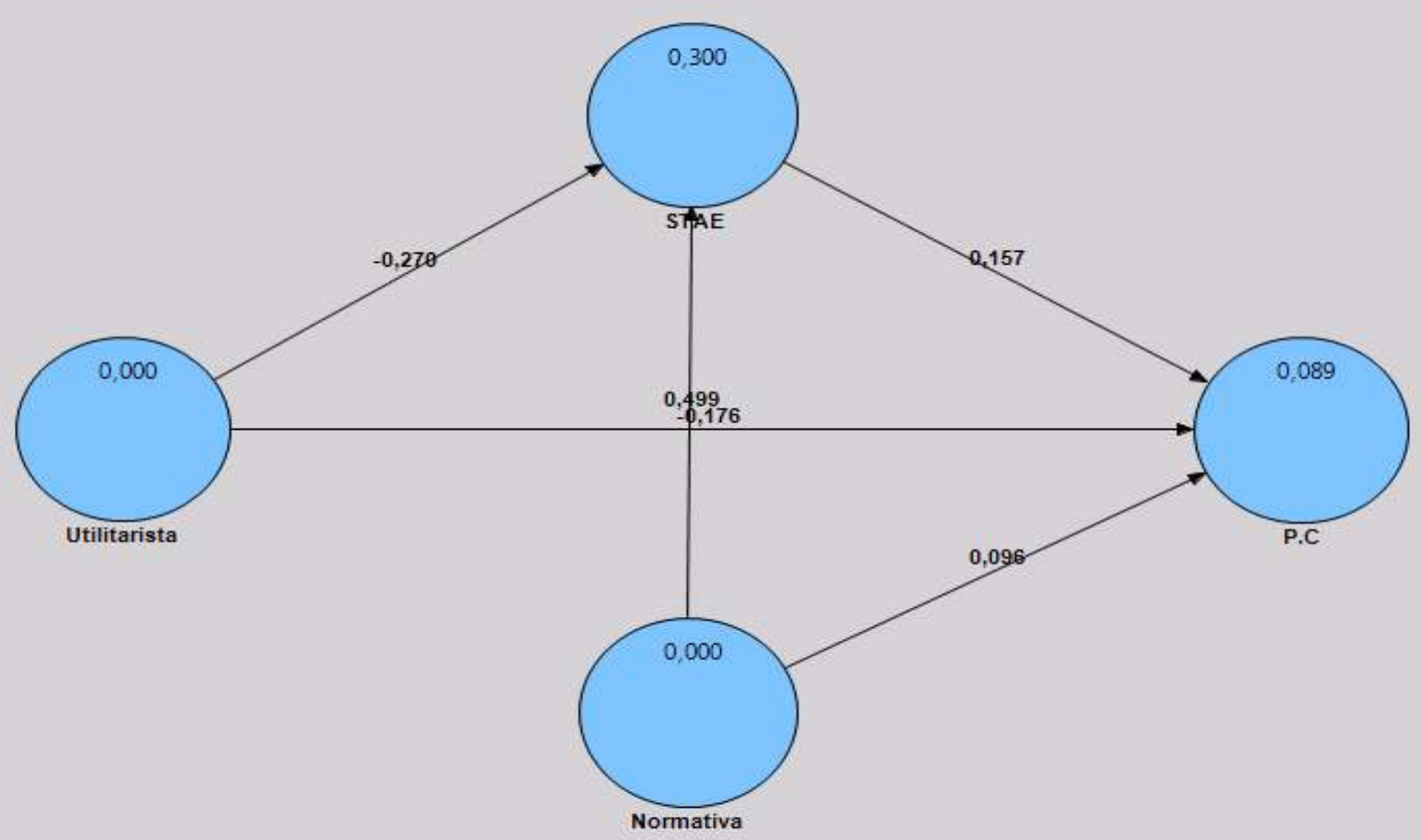

Fonte: SmartPLS 2.0

Observou-se que a Identidade Organizacional (construto utilitarista e normativo) explica 30\% (coeficiente igual a 0,300) dos Sistemas de Alto Envolvimento, sendo considerado um efeito de tamanho médio. Há de se levar em conta, entretanto que o construto utilitarista possui correlação negativa $(-0,270)$ com os STAES, isso é, os valores crescentes de utilitarismo levam a menores índices de STAE. Já o construto normativo possui uma correlação positiva $(0,499)$ demonstrando que, na medida em que se eleva o caráter normativo, elevam-se os índices de STAE nas organizações.

Em relação ao Potencial de Crescimento, observa-se que a Identidade Organizacional, juntamente com os STAE explicam $8,90 \%$ do potencial de crescimento das MPE's. Deve-se, entretanto, perceber que:

- O caráter utilitarista impacta negativamente o Potencial de Crescimento $(-0,176)$;

- O caráter normativo impacta positivamente o Potencial de Crescimento $(0,096)$;

- Os STAES possuem maior influência sobre o Potencial de Crescimento que as outras variáveis latentes, tendo uma correlação de 0,157 , sendo considerado um tamanho de efeito pequeno, mas significante dentro dos estudos das ciências sociais.

A fim de comprovar, ainda mais, a consistência dessas informações, foi realizada uma análise da correlação entre as variáveis, utilizando-se do teste de Pearson - Tabela 6. 
Tabela 2 - Correlações entre as Variáveis IO, STAE e PC

\begin{tabular}{|c|c|c|c|c|c|}
\hline \multirow{2}{*}{\multicolumn{2}{|c|}{ Variáveis }} & \multicolumn{2}{|l|}{ I.O. } & \multirow{2}{*}{ STAE } & \multirow{2}{*}{ P.C. } \\
\hline & & Utilitarista & Normativa & & \\
\hline \multirow{3}{*}{ Utilitarista } & Pearson Correlation & 1,000 & & & \\
\hline & Sig. (2-tailed) & & & & \\
\hline & $\mathrm{N}$ & 109 & & & \\
\hline \multirow{3}{*}{ Normativa } & Pearson Correlation & 0,097 & 1,000 & & \\
\hline & Sig. (2-tailed) & 0,317 & & & \\
\hline & $\mathrm{N}$ & 109 & 109 & & \\
\hline \multirow{3}{*}{ STAE } & Pearson Correlation & $-0,071$ & $0,475^{\star * *}$ & 1,000 & \\
\hline & Sig. (2-tailed) & 0,462 & 0,000 & & \\
\hline & $\mathrm{N}$ & 109 & 109 & 109 & \\
\hline \multirow{3}{*}{ P.C. } & Pearson Correlation & $-0,030$ & $0,222^{* *}$ & $0,263^{* * *}$ & 1,000 \\
\hline & Sig. (2-tailed) & 0,753 & 0,021 & 0,006 & \\
\hline & $\mathrm{N}$ & 109 & 109 & 109 & 109 \\
\hline
\end{tabular}

Nota: * p-valor $<10 \%$, ${ }^{* *} p$-valor $<5 \%,{ }^{* * *} p$-valor $<1 \%$

Fonte: elaborada pelo autor, com base nos resultados da pesquisa.

Os resultados confirmam os dados gerados na análise das influências entre as variáveis latentes do modelo estrutural na Modelagem de Equações Estruturais (MEE), isto é, verifica-se que há correlação positiva do caráter normativo da Identidade Organizacional com o nível de $\operatorname{STAE}(0,475)$ e com o Potencial de Crescimento $(0,222)$, contribuindo para a comprovação da hipótese $\mathrm{H}_{1 a}$ que afirma que o nível de adoção dos Sistemas de Trabalho de Alto Envolvimento nas empresas, medido por meio da escala de Foreman e Whetten (2002) será maior nas organizações com identidade normativa na percepção de seus proprietários, medida conforme Ciavarelha (2003). A identidade normativa assemelha-se às estratégias Soft e que focam na participação e comprometimento dos colaboradores de uma organização.

Correlações positivas, também, entre o nível de STAE e o potencial de crescimento $(0,263)$ corroborando para comprovação da hipótese $\mathrm{H}_{2}$, isto é, quanto maior o nível de adoção de Sistemas de Trabalho de Alto Envolvimento, mensurado pela escala de Foreman e Whetten (2002), maior será o potencial de crescimento das empresas, mensurado pela escala de Rutherford et al. (2004).

Por fim, verificou-se correlações negativas do caráter utilitarista de 10 com o STAE $(-0,710)$ e o Potencial de Crescimento $(-0,310)$, auxiliando como indicador comprobatório das seguintes hipóteses:

- $\mathrm{H}_{1 b}$, revelando que o nível de adoção dos Sistemas de Trabalho de Alto Envolvimento nas empresas, medido pela escala de Foreman e Whetten (2002) será menor nas organizações com identidade utilitarista na percepção de seus proprietários, medida conforme Ciavarelha (2003). Esse tipo de identidade assemelha-se às estratégia Hard de gestão de Pessoas, cujo foco está no rígido controle dos colaboradores.

- $\mathrm{H}_{3}$, onde se afirma que o tipo de identidade organizacional (normativa ou utilitarista) está relacionado ao maior ou menor potencial de crescimento das empresas, tendo o nível de adoção dos sistemas de trabalho de alto envolvimento como mediador. 
O perfil dos micro e pequenos empresários investigados revelou que $66,11 \%$ são do sexo masculino, demonstrando, ainda, a predominância do gênero masculino na abertura de novas empresas no Brasil, conforme dados do Sebrae (2012). Dos pesquisados, 57,8\% possuem nível superior, $56 \%$ possuem de 21 a 40 anos, indicando que o número de jovens e adultos com maior nível educacional prevalece na amostra em estudo. $45,8 \%$ se consideram apenas sócios, proprietários ou representantes da alta direção, os demais alegram ocupar, também, algum cargo de gestão dentro das MPE's, corroborando com a hipótese de que, na etapa do Ciclo de Vida Organizacional (CVO) onde essas empresas se enquadram, exige-se a presença do proprietário como dono e como gestor (MOUNT, 1993). Essa informação é complementada com o fato de que $51,4 \%$ das empresas tem até 10 anos de operação que, na visão de Miller e Friesen (1984), elas são enquadradas na fase de crescimento do CVO, cuja presença do sócio-proprietário se faz necessária. 82,6\% das empresas encontram-se nos setores de comércio e serviços, indo a favor dos dados recentes da economia do Brasil que indica o crescimento nesses setores (SEBRAE, 2011).

A investigação da Identidade Organizacional, categorizada em dois carácteres, na percepção dos proprietários, indicou que $51,2 \%$ da amostra possuem o caráter utilitarista, muito próximo do caráter normativo. As empresas utilitaristas concentraram-se, em relação à quantidade, nos setores da construção civil e de serviços, entre os que ocupam cargos de gestão e em empresas com mais de 10 anos de operação. Já as empresas classificadas como normativas predominaram nos setores de comércio e serviços, entre os que tinham mais de 50 anos, em empresas com até 10 anos de operação e entre os que ocupavam a função de sócios. Não se observaram diferenças entre os carácteres quando analisados as relações com a questão de gênero, escolaridade e no setor comercial, sugerindo que novas pesquisas devam ser realizadas, principalmente no viés qualitativo para buscar o entendimento desses resultados.

Observou-se um nível maior de STAE para os indivíduos do sexo masculino, com mais de 40 anos, com graduação, que atuavam como sócios, principalmente nos setores de comércio e serviços. Os dados revelaram, ainda, que um número maior de empresas do tipo normativas possuíam maiores níveis de STAE, corroborando para confirmação da hipótese $\mathrm{H}_{1 a}$, de que empresas com esse caráter, possuem maior nível de STAE.

A análise do Potencial de Crescimento revelou que não foi possível haver distinção entre gênero. As empresas classificadas como "em crescimento" possuíram maiores concentrações entre aqueles que possuíam mais de 50 anos, graduados e pós-graduados e que atuavam como sócios em empresas dos setores da construção civil e de serviços. Isso sugere que o potencial de crescimento pode estar relacionado com o nível de amadurecimento dos indivíduos e com o grau de escolaridade dos mesmos. Observou-se, ainda, que as empresas normativas possuem maiores concentrações de empresas em crescimento, corroborando com a hipótese $\mathrm{H}_{3}$ de que a Identidade tem impacto sobre o potencial de crescimento das MPE's.

Foi analisado, ainda, as empresas consideradas de alto crescimento, isso é, aquelas com mais de 10 funcionários no ano inicial de investigação e que tiveram um crescimento de $20 \%$ da mão de obra nos últimos três anos analisados. Foram encontradas apenas 8 empresas nessa categoria. A análise, portanto, revelou que os resultados são inconclusivos em relação ao caráter de IO, uma vez que o número de empresas foi exatamente igual nos dois carácteres. Revelou que elas possuem um médio nível de STAE. Contudo, esses 
dados amostrais não são relevantes para generalização à população, carecendo de maiores pesquisas para confirmação dos resultados em empresas dessa categoria.

\section{CONSIDERAÇÕES FINAIS}

O estudo tem limitações de teoria, método, público e pela impossibilidade de generalização. Os dados não podem ser generalizados a todas as Micro e Pequenas Empresas uma vez que a amostragem foi do tipo não probabilística.

A teoria de Sistemas de Trabalho de Alto Envolvimento ainda está se iniciando no Brasil, necessitando de maiores pesquisas para analisar os construtos envolvidos na estruturação e mensuração desses sistemas.

O método tem suas limitações em função de viesses tanto do pesquisador quanto dos respondentes. O fato de o questionário ser online dificultava o controle das respostas. Aliado ao fator tempo, onde o respondente levava um tempo médio de resposta em torno de 20 minutos, podem ter alterado algumas questões. Como exemplo muitas questões não foram respondidas ou foram parcialmente. Outra limitação do método foi a utilização da análise quantitativa que dificulta o entendimento e aprofundamento de algumas dúvidas surgidas durante a análise de dados, revelando a necessidade de investigações qualitativas.

Outro cuidado é que, na análise do potencial de crescimento, foi considerada a percepção dos proprietários. Tentou-se minimizar o impacto dessa análise, solicitando, aos respondentes, que informassem o faturamento real. Entretanto, apenas 59 empresas responderam a essa questão. Ela foi considerada, apenas, para balizar o percentual de crescimento informado pelos proprietários de MPE's.

O público escolhido também é um fator limitante da pesquisa. Devido à extensa área territorial, o número de micro e pequenas empresas participantes representam um percentual muito pequeno quando comparado ao universo dessas empresas. Outro fator limitante é a classificação do porte da empresa, que possuem diferentes denominações e o fato de que não se buscou equalizar, na coleta de dados, um número igual de empresas por porte.

Essas limitações abrem possibilidades para estudos futuros e maior compreensão dos temas de Identidade Organizacional, Sistemas de Trabalho de Alto Envolvimento e Potencial de Crescimento das organizações.

Esse estudo poderá contribuir para uma melhor gestão nas MPE's, através da sugestão de um modelo baseado em uma identidade normativa e em práticas de trabalho que envolva os funcionários com o objetivo de buscar o crescimento dessas empresas.

$\mathrm{Na}$ academia abre um novo leque de oportunidades de estudos no intuito de aprofundar as teorias sobre IO, STAE e PC no Brasil, assim como compreender como elas se instauram e se desenvolvem diferentes contextos econômicos. Sugere-se pesquisar tais relações em setores econômicos específicos e por porte da empresa, como por exemplo, empresas de médio porte no setor de empresas de alta tecnologia, empresas do terceiro setor, empresas agrícolas, dentre outras tantas possibilidades. Posteriormente seria possível comparar se os resultados da relação entre IO STAE e PC se mantêm ou se alteram em relação a esses setores.

Sugere-se, ainda, a realização de pesquisas qualitativas como meio de aprofundar e fundamentar os achados de pesquisa. Pode-se, também, realizar estudos longitudinais quali-quantitativos visando analisar as 
variações de crescimento dessas empresas em relação aos construtos de Identidade Organizacional e dos Sistemas de Trabalho de Alto Envolvimento.

E, por fim, sugere-se correlacionar outras variáveis ao modelo conceitual, como analisando, por exemplo, o nível de comprometimento organizacional e seu impacto nos STAE e no potencial de crescimento. Outras variáveis possíveis são: Entrincheiramento, Valores e Clima Organizacionais, tudo isso visando alinhar os estudos de comportamento organizacional às estratégias de Gestão de Pessoas.

\section{REFERÊNCIAS}

ALBERT, S.; WHETTEN, D. A. Organizational Identity. In: L. . Climmings; B. . Staw (Eds.); Research in Organizational Behavior, 1985. Greenwich: Jay Press.

ALBUQUERQUE, L. G. A Gestão Estratégica de Pessoas. In: M. T. L. Fleury (Ed.); As Pessoas na Organização, 2002. Rio de Janeiro: Gente.

BECKER, B. E.; HUSELID, M. A.; ULRICH, D. Gestão Estratégica de Pessoas com Scorecard: Interligando Pessoas, Estratégia e Performance. Rio de Janeiro: Campus, 2001.

CIAVARELLA, M. A. The adoption of high-involvement practices and processes in emergent and developing firms: A descriptive and prescriptive approach. Human Resource Management, v. 42, n. 4, p. 337-356, 2003. Disponível em: <http://doi.wiley.com/10.1002/hrm.10094>. Acesso em: 23/7/2011.

COHEN, J. Statistical Power Analysis for The Behavioral Sciences. New York: Academic Press, 1977.

COMBS, J. G.; CROOK, T. R.; SHOOK, C. L. The dimension of organizational performance and its implications for strategic management research. In: D. J. KETCHEN; D. D. BERGH (Eds.); Research methodology in strategy and management. p.259-286, 2005. San Diego: Elsevier.

DAVIDSSON, P.; ACHTENHAGEN, L.; NALDI, L. Research on Small Firm Growth : A Review. International Business, 2009 .

DOODY, S. P. High-Involvement Work Systems : Their Effect on Employee Turnover and Organisational Performance in New Zealand Organisations. Lincoln University - Thesis Doctoral, 2007.

FABI, B.; RAYMOND, L.; LACOURSIÈRE, R. Strategic alignment of HRM practices in manufacturing SMEs: a Gestalts perspective. Journal of Small Business and Enterprise Development, v. 16, n. 1, p. 7-25, 2009.

FAEDPYME. Análisis estratégico para el desarrollo de la MPYME en Iberoamérica: Brasil. 2009.

FREIRE, D. A. L. Identidade Organizacional, Estratégia de Gestão de Pessoas e o Potencial de Crescimento em Empresas de Micro e Pequeno Porte, 2013. Universidade Nove de Julho.

GIOIA, D. A; THOMAS, J. B. Identity, Image, and Isssue Interpretation: Sensemaking during Strategic Change in Academia. Administrative Science Quarterly, v. 41, p. 370-403, 1996.

IBGE. Demografia das Empresas 2008. IBGE, 2010.

LAWLER, E. E. Strategic pay: Aligning or- ganizational strategies and pay systems. San Francisco: Jossey-Bass, 1990. LEGGE, K. HRM: Rhetorics and Realities. Basingstoke: Macmillan Business., 1995.

LIMA, E. Estratégia de Pequenas e Médias Empresas: Uma Revisão. ReGE, v. 17, n. 2, p. 169-187, 2010.

LLOYD, C.; PAYNE, J. The Only Show in Town (If a Pretty Pathetic one at that): Re-Evaluatin the High-Performance Workplace as a Vehicle for the Uk high skills Project. International Labour Process. Anais... , 2004. Amsterdam.

NADLER, D. A.; GERSTEIN, M. S.; SHAW, R. Arquitetura Organizacional: A Chave para a Mudança Empresarial. Rio de Janeiro: Campus, 1994.

PASANEN, M. SME Growth Strategies: A Comparison of Young and Long-Lived Firms. International conference on Business and Information, p. 12 - 14, 2006.

PINSONNEAULT, A.; KRAEMER, K. L. Survey Research in Management Information Systens: An Assessement. Journal of Management Information System, 1993.

PRATT, M. G.; FOREMAN, P. O. Identity Dialogues. Academy of Management Review, v. 25, n. 1, p. 141-152, 2000.

Disponível em:

<http://www.pubmedcentral.nih.gov/articlerender.fcgi?artid=3208207\&tool=pmcentrez\&rendertype=abstract>.

RINGLE, C. .; WILL, S. SmartPLS 2.0 (M3) Beta. Disponível em: <http://www.smartpls.de>. Acesso em: 6/7/2012.

RUAS, R. L. Novos princípios gerenciais e a organização e gestão do trabalho. CNI/SESI, 1995. 
RUTHERFORD, M. W.; BULLER, P. F.; MCMULLEN, P. R. Human resource management problems over the life cycle of small to medium-sized firms. Human Resource Management, v. 42, n. 4, p. 321-335, 2004. Disponível em: <http://doi.wiley.com/10.1002/hrm.10093>. Acesso em: 14/8/2011.

SEBRAE; DIEESE. Anuário do Trabalho na Micro e Pequena Empresa: 2009. 2010.

SEBRAE/SP. Doze anos de Monitoramento da Sobrevivência e Mortalidade de Empresas. SEBRAE/SP, p. 1-51, 2010.

STOREY, J. Developments in the management of human resources: An interim report. Warwick Papers in Industrial Relation, v. 17, 1987.

TOCHER, N.; RUTHERFORD, M. W. Perceived acute human resource management problems in small and medium firms: an empirical examination. Entrepreneurship Theory and Practice, v. 3, n. 334, p. 455-480, 2009.

VENKATRAMAN, N.; RAMANUJAM, V. Measurement of business performance in strategy research: a comparison of approaches. Academy of Management Review, v. 11, n. 4, p. 801-814, 1986.

WALTON, R. E. From Control to Commitment in The Workplace. Harvard Business Review, 1985. 